\title{
Comparison of different induction agents used for rapid sequence intubation
}

\author{
Enass Farouk Aboshoushah ${ }^{1 *}$, Aisha Yahya Saddeek², Wail Mohammad Alanazi, \\ Mohammed Abdulaziz Alghamdi ${ }^{4}$, Raneem Abdulhamid Alrahwan ${ }^{4}$, Nojoud Adnan Khayat ${ }^{5}$, \\ Muqrin Safar Alotaibi ${ }^{6}$, Khaled Nasser Alharbi ${ }^{7}$, Ahmed Mohammed Kurdi ${ }^{8}$, \\ Shouq Sulaiman Alrumayh', Rimaz Mohammed Alassiri ${ }^{10}$
}

\author{
${ }^{1}$ Department of Anaesthesia, King Abdulaziz Hospital, Jeddah, Saudi Arabia \\ ${ }^{2}$ Department of Intensive Care Unit, King Fahad Hospital, Medina, Saudi Arabia \\ ${ }^{3}$ Department of Pharmacy, Prince Mutaib bin Abdulaziz Hospital, Sakaka, Saudi Arabia \\ ${ }^{4}$ College of Medicine, Ibn Sina National College, Jeddah, Saudi Arabia \\ ${ }^{5}$ Department of Pharmacy, Al Noor Specialist Hospital, Mecca, Saudi Arabia \\ ${ }^{6}$ College of Medicine, Shaqra Medical University, Aldawadmi, Saudi Arabia \\ ${ }^{7}$ Department of Emergency Medicine, Imam Abdulrahman Al Faisal Hospital, Riyadh, Saudi Arabia \\ ${ }^{8}$ Department of Emergency Medicine, King Salman Medical City, Medina, Saudi Arabia \\ ${ }^{9}$ College of Medicine, Qassim University, Qassim, Saudi Arabia \\ ${ }^{10}$ College of Medicine, Umm Al-Qura University, Mecca, Saudi Arabia
}

Received: 28 August 2021

Accepted: 02 September 2021

\section{*Correspondence:}

Dr. Enass Farouk Aboshoushah,

E-mail: enass.farouk@yahoo.com

Copyright: ( $)$ the author(s), publisher and licensee Medip Academy. This is an open-access article distributed under the terms of the Creative Commons Attribution Non-Commercial License, which permits unrestricted non-commercial use, distribution, and reproduction in any medium, provided the original work is properly cited.

\begin{abstract}
Securing the airway is the first step that clinicians take care of for clinically unstable patients. Rapid sequence intubation (RSI) has been reported by many clinicians to effectively achieve this. Many agents have been reported in the literature as effective induction agents for RSI. In this literature review, we have discussed the efficacies of the different induction agents that are commonly reported in the literature for RSI. Furthermore, RSI is done by paralytic and sedative agents that rapidly render the patient flaccid and unconscious to facilitate the emergent approaches to achieve successful tracheal intubation and minimize adverse events. We have discussed the efficacies and adverse events of benzodiazepines and barbiturates, ketamine, propofol, and etomidate. Each of these modalities has its advantages and adverse events, and clinicians should choose what is best for their patients based on the aforementioned discussion for each drug modality. We recommend that further investigations might be needed for further optimization of the induction agent and the relevant doses.
\end{abstract}

Keywords: Induction agents, Rapid sequence intubation, Benzodiazepines, Propofol, Etomidate

\section{INTRODUCTION}

Securing the airway is the first step that clinicians take care of for clinically unstable patients. Rapid sequence intubation (RSI) has been reported by many clinicians to effectively achieve this. This necessitates the use of an induction agent and a neuromuscular blocker to successfully achieve successful intubation procedures with favorable outcomes. ${ }^{1-3}$ Using these drugs rapidly renders the patient flaccid and unconscious which enables the 
attending clinicians to achieve rapid and effective intubations, and reduce the risk of aspiration. Accordingly, a successful RSI procedure might hugely depend on choosing the right induction agents. Many agents have been reported in the literature as effective induction agents for RSI. Although paralytic agents can be used to achieve successful intubation. The process is done without sedation. Therefore, the patient is usually aware of the surrounding atmosphere, including the potentially significant pain that might occur during intubation. However, no response can be obtained from the patient, which might subject him to several consequences and worsen the outcomes. ${ }^{4,5}$ In addition, manipulating the airways might significantly lead to the development of significant adverse events as a result of the potential psychological response. Consequently, hypertension, tachycardia, and increased intracranial pressure might develop. ${ }^{6}$ In this literature review, we aim to discuss the efficacies of the different induction agents that are commonly reported in the literature for RSI.

\section{METHODS}

This literature review is based on an extensive literature search in Medline, Cochrane, and EMBASE databases which was performed in August 2021 using the medical subject headings $(\mathrm{MeSH})$ or a combination of all possible related terms. ${ }^{7,8}$ This was followed by the manual search for papers in Google Scholar while the reference lists of the initially included papers. ${ }^{9,10}$ Papers discussing the induction agents used for rapid sequence intubation were screened for relevant information, with no limitation placed on date, language, age of participants, or publication type.

\section{DISCUSSION}

Using induction agents is very important to effectively achieve rapid sequence intubation. ${ }^{11}$ Using these modalities in such situations has been reported with improved intubation conditions, a blunt sympathetic response, and amnesia. Thus, they can significantly reduce or even prevent the development of the severe adverse events that might be associated with using paralytic agents, as a result of the sedation component of these modalities. Ameliorating the underlying conditions of the patient. In addition, the main functions in achieving successful RSI can also be achieved by these modalities, as indicated by previous investigations for some induction agents. For instance, Denmark et al previously reported that ketamine was successfully used to decrease bronchospasm in patients suffering from severe asthma. ${ }^{12}$ Enhanced laryngoscopic view during the intubation process might also be better with using the induction agents. Therefore, this can enhance the associated outcomes and furtherly facilitate the underlying procedures. This is usually important because laryngoscopic are commonly performed within the earliest periods of neuromuscular paralysis. This is achievable because of the paralytic characteristics of these modalities, the associated sedation also aids in achieving complete relaxation of the underlying muscles. Therefore, can significantly improve laryngoscopy and the associated outcomes. ${ }^{11,13}$ This has been indicated by a previous investigation that concluded that even some paralytic agents can induce a complete neuromuscular paralysis and blocking, using induction agents and sedatives is still recommended to enhance the intubation outcomes. ${ }^{11}$ Accordingly, this section discusses some of the most commonly reported induction agents that have been used to achieve RSI.

\section{Benzodiazepines and barbiturates}

Amnesia and sedation can be obtained from using benzodiazepines because they have a marked effect on gamma-aminobutyric acid (GABA) receptors. The most commonly reported drug for RSI is midazolam, which is the most rapid modality that acts within 30-60 seconds after administration and lasts for 15-30 minutes. ${ }^{14,15}$ However, it should be noted that midazolam and other benzodiazepines do not have analgesic properties but they have anticonvulsant effects. Therefore, they are recommended for RSI in patients suffering from status epilepticus. It has been estimated that moderate hypotension and a potential reduction in the mean arterial blood pressure can occur after the administration of midazolam in $10-25 \%$ of the patients. ${ }^{14,15}$ Accordingly, midazolam should be contraindicated in cases of shock and hypovolemia. However, lowering the dose might intervene against the development of such conditions without impairing the intubation outcomes. However, this should only be approached in cases when other substitutes are not available, as in such cases ketamine, or etomidate are suggested because of their favorable effects on the patient's hemodynamics. Underdosing might be another issue when using midazolam, and previous studies have demonstrated that midazolam intravenous infusions can be used for sedation and intubation in many cases. ${ }^{15,16}$ Although diazepam and lorazepam can be effectively used for long-term sedation, they are not recommended for RSI as they might induce toxicity. ${ }^{17}$ Although barbiturates are no longer widely available, some of them are still used, as thiopental sodium and methohexital. However, they do not have analgesic effects. Suppressed neuronal activities can be obtained by using thiopental.

As a result, it can be used for patients suffering from conditions that elevate the intracranial tension. However, the drug can inversely lead to hypotension because it is a potent vasodilator. This can also lead to reduced cerebral perfusion. Therefore, dose consideration is essential to achieve better outcomes. Another contraindication of thiopental is the presence of an underlying respiratory disease because it might cause bronchospasm as a result of histamine release. ${ }^{18}$ Besides, barbiturates have been reported to have potential immunosuppressive effects. Consequently, they should not be used in cases of intubation for sepsis. ${ }^{19-22}$ 


\section{Etomidate}

Etomidate is a hypnotic-sedative agent that has been successfully used for RSI, being derived from imidazole. It mainly inhibits GABA leading to reduced neurotransmission and excitability and inducing anesthesia. Intravenous administration of the drug can maintain an action for 3-12 minutes to successfully conduct RSI. ${ }^{23}$ No histamine release and no hemodynamic changes have been observed with the drug, as compared to the aforementioned modalities, making this drug an ideal candidate for patients with hypotension and elevated intracranial pressure. ${ }^{24-26}$ However, it should be recommended that in cases of etomidate administration, an analgesic modality should be administered priorly (as opioids) in patients suffering from cardiovascular and respiratory diseases. It is because of the absent analgesic effect of etomidate. Thus, no noxious effects inhibition during the process of intubation, which can induce some adverse events. ${ }^{27}$ However, it should be noted that etomidate has been reported with many adverse events and complications that might reduce the prognosis of the patients. These mainly include myoclonus, adrenal suppression, and regional brain excitation following intubation. ${ }^{28,29}$ Therefore, the administration of this drug should be contraindicated in patients at increased risk of developing these disorders. Besides, it is recommended that benzodiazepines or propofol should be coadministered to enhance post-intubation sedation, maintaining adequate neuronal inhibition.

Etomidate can significantly lead to a reversible state of adrenal suppression because it can reversibly inhibit 11beta-hydroxylase, leading to decreased production and synthesis of cortisol. However, it should be noted that cortisol levels do not decrease below the normal range levels, and this reduction does not usually persist for long periods. Accordingly, when drawing a clinical decision for patients with sepsis, the administration of etomidate should be based on a wise balance between the favorable effects on the patient's hemodynamics and the unfavorable effects on the adrenal glands. Besides, it is recommended that etomidate should not be administered as a boosting dose or as an infusion. Some authors suggested that glucocorticoids should be administered with etomidate for patients managed for sepsis. However, the evidence regarding this suggestion is not evident, and further investigations are needed. ${ }^{30,31}$

\section{Ketamine and propofol}

Ketamine is a favorable drug to induce RSI because it has been reported to cause amnesia, sedation, and analgesia. Besides, the mechanism of action of this drug modality involves the interaction with various receptors including GABA, and opioid receptors leading to autoinhibition and analgesia, respectively. ${ }^{32}$ Besides, it increases the release of catecholamines leading to a significant impact on the heart rate, blood pressure, and cerebral blood flow. ${ }^{33,34}$ In addition to actions, it also inhibits nicotinic receptors and reduces the release of nitric oxide. ${ }^{35}$ Another advantage of the modality is that it acts quickly, which makes it a good option for performing rapid intubation processes. Although the drug has been reported with minimal effects on hemodynamics, it has been reported that it should be administered with caution when performing RSI for patients with impaired catecholamines secretion and impaired sympathetic activities. ${ }^{33,34}$ Previous studies have also demonstrated that ketamine might be associated with enhanced reperfusion outcomes on the myocardium. ${ }^{33,34}$ However, clinicians should be cautious when considering it for patients with coronary heart diseases to preventing the development of ischemia. Some patients might experience the reemergence phenomenon. However, this probably does not occur with the dose that is used for RSI, and most probably due to the administration of a sedative dose, or due to the co-administration of benzodiazepines. ${ }^{36}$ There have been some concerns about the administration of ketamine for patients suffering from head injuries due to the potential elevation in the intracranial pressure. Although it has been indicated that ketamine might be associated with increased release of catecholamines, other investigations showed that this can be neutralized by the co-administration of GABA agonists. ${ }^{37-39}$ Moreover, the use of ketamine might enhance neurological outcomes by improving the process of reperfusion. ${ }^{34,39} \mathrm{~A}$ meta-analysis by Cohen et al indicated that ketamine administration was not significantly associated with adverse events and neurological complications. ${ }^{40}$

Propofol has also been reported to be effectively used for RSI, as an agent that can induce amnesia and sedation, being an alkylphenol-derivative, and highly lipid-soluble acting on the GABA receptor complex. The drug interferes with the process of long-term memory development, leading to amnesia, and directly suppresses brain activities leading to sedation. ${ }^{41,42}$ No significant differences were noticed among the different populations. ${ }^{43,44}$ However, the drug takes more time to peak within the serum of children. ${ }^{45}$ The drugs have also been observed to reduce airway resistance, and therefore, it is recommended for patients with bronchospasm. ${ }^{27,46}$ Another advantage of the drug is that it might be used for patients with brain-related disorders because it has a significant neuroinhibitory effect. However, it should be used indicated for hemodynamically stable patients only as it might cause peripheral vasodilatation and myocardial depression. ${ }^{47}$ Besides, it has been indicated that QT interval is not prolonged following the drug administration. ${ }^{48}$ However, serum lipase and triglycerides levels elevate following the administration of propofol infusions. ${ }^{49}$ Allergic reactions are becoming rare with the new generations of the drug. It is worth mentioning that ketofol, a combination of ketamine and propofol, is used by many clinicians to induce RSI. This is done to obtain the favorable effects of each modality, including the analgesic characteristics of ketamine and reducing the hypotensive characteristics of propofol. Besides, they are very useful for patients with bronchospasm because both of the drug modalities are potent bronchodilators. Not many investigations have 
assessed the efficacy of ketofol and the reported sedation effect seems to be significant, however, it comes with a hypotensive effect that is, fortunately, less than that reported with propofol alone. ${ }^{50}$

\section{CONCLUSION}

Rapid sequence intubation is done by paralytic and sedative agents that rapidly render the patient flaccid and unconscious to facilitate the emergent approaches to achieve successful tracheal intubation and minimize adverse events. We have discussed the efficacies and adverse events of benzodiazepines and barbiturates, ketamine, propofol, and etomidate. Each of these modalities has its advantages and adverse events and clinicians should choose what is best for their patients based on the aforementioned discussion for each drug modality. We recommend that further investigations might be needed for further optimization of the induction agent and the relevant doses.

\section{Funding: No funding sources \\ Conflict of interest: None declared \\ Ethical approval: Not required}

\section{REFERENCES}

1. Sagarin MJ, Chiang V, Sakles JC. Rapid sequence intubation for pediatric emergency airway management. Pediatric emergency care. 2002;18(6):417-23.

2. Sakles JC, Laurin EG, Rantapaa AA, Panacek EA. Airway management in the emergency department: a one-year study of 610 tracheal intubations. Annals of emergency medicine. 1998;31(3):325-32.

3. Tayal VS, Riggs RW, Marx JA, Tomaszewski CA, Schneider RE. Rapid-sequence intubation at an emergency medicine residency: success rate and adverse events during a two-year period. Academic emergency medicine : official journal of the Society for Academic Emergency Medicine. 1999;6(1):31-7.

4. Ballard N, Robley L, Barrett D, Fraser D, Mendoza I. Patients' recollections of therapeutic paralysis in the intensive care unit. American journal of critical care : an official publication, American Association of Critical-Care Nurses. 2006;15(1):86-94.

5. Playfor SD, Thomas DA, Choonara II. Recall following paediatric intensive care. Paediatric anaesthesia. 2000;10(6):703-4.

6. Sivilotti ML, Ducharme J. Randomized, double-blind study on sedatives and hemodynamics during rapidsequence intubation in the emergency department: The SHRED Study. Annals of emergency medicine. 1998;31(3):313-24.

7. Ghozy S, Tran L, Naveed S. Association of breastfeeding status with risk of autism spectrum disorder: A systematic review, dose-response analysis and meta-analysis. Asian J Psychiatr. 2020;48:101916.
8. Mahmoud AR, Dahy A, Dibas M, Abbas AS, Ghozy S, El-Qushayri AE. Association between sarcoidosis and cardiovascular comorbidity: A systematic review and meta-analysis. Heart Lung. 2020;49(5):512-7.

9. Ghozy S, Nam NH, Radwan I. Therapeutic efficacy of hepatitis B virus vaccine in treatment of chronic HBV infections: A systematic review and metaanalysis. Rev Med Virol. 2020;30(3):e2089.

10. Hashan MR, Ghozy S, El-Qushayri AE, Pial RH, Hossain MA, Al Kibria GM. Association of dengue disease severity and blood group: A systematic review and meta-analysis. Rev Med Virol. 2021;31(1):1-9.

11. Sivilotti ML, Filbin MR, Murray HE, Slasor P, Walls RM. Does the sedative agent facilitate emergency rapid sequence intubation? Academic emergency medicine : official journal of the Society for Academic Emergency Medicine. 2003;10(6):612-20.

12. Denmark TK, Crane HA, Brown L. Ketamine to avoid mechanical ventilation in severe pediatric asthma. The Journal of emergency medicine. 2006;30(2):163-66.

13. El-Orbany MI, Wafai Y, Joseph NJ, Salem MR. Does the choice of intravenous induction drug affect intubation conditions after a fast-onset neuromuscular blocker? Journal of clinical anesthesia. 2003;15(1):9-14.

14. Blumer JL. Clinical pharmacology of midazolam in infants and children. Clinical pharmacokinetics. 1998;35(1):37-47.

15. Nordt SP, Clark RF. Midazolam: a review of therapeutic uses and toxicity. The Journal of emergency medicine. 1997;15(3):357-65.

16. Sagarin MJ, Barton ED, Sakles JC, Vissers RJ, Chiang V, Walls RM. Underdosing of midazolam in emergency endotracheal intubation. Academic emergency medicine : official journal of the Society for Academic Emergency Medicine. 2003;10(4):32938.

17. Wilson KC, Reardon C, Theodore AC, Farber HW. Propylene glycol toxicity: a severe iatrogenic illness in ICU patients receiving IV benzodiazepines: a case series and prospective, observational pilot study. Chest. 2005;128(3):1674-81.

18. Gholipour Baradari A, Firouzian A, Zamani Kiasari A. Effect of Etomidate Versus Combination of Propofol-Ketamine and Thiopental-Ketamine on Hemodynamic Response to Laryngoscopy and Intubation: A Randomized Double Blind Clinical Trial. Anesthesiology and pain medicine. 2016;6(1):e30071.

19. Meço BC, Bermede AO, Alanoğlu Z, Yaka O, Alkış N. Influence of Different Doses of Ketamine on Intubating Conditions during a Rapid Sequence Induction and Intubation Model. Turkish journal of anaesthesiology and reanimation. 2016;44(1):26-31.

20. Russo H, Bressolle F. Pharmacodynamics and pharmacokinetics of thiopental. Clinical pharmacokinetics. 1998;35(2):95-134. 
21. Reich DL, Hossain S, Krol M. Predictors of hypotension after induction of general anesthesia. Anesthesia and analgesia. 2005;101(3):622-8.

22. Ploppa A, Kiefer RT, Nohé B. Dose-dependent influence of barbiturates but not of propofol on human leukocyte phagocytosis of viable Staphylococcus aureus. Crit Care Med. 2006;34(2):478-83.

23. Bergen JM, Smith DC. A review of etomidate for rapid sequence intubation in the emergency department. The Journal of emergency medicine. 1997;15(2):221-30.

24. Benson M, Junger A, Fuchs C, Quinzio L, Böttger S, Hempelmann G. Use of an anesthesia information management system (AIMS) to evaluate the physiologic effects of hypnotic agents used to induce anesthesia. Journal of clinical monitoring and computing. 2000;16(3):183-90.

25. Guldner G, Schultz J, Sexton P, Fortner C, Richmond $\mathrm{M}$. Etomidate for rapid-sequence intubation in young children: hemodynamic effects and adverse events. Academic emergency medicine : official journal of the Society for Academic Emergency Medicine. 2003;10(2):134-9.

26. Sokolove PE, Price DD, Okada P. The safety of etomidate for emergency rapid sequence intubation of pediatric patients. Pediatric emergency care. 2000;16(1):18-21.

27. Eames WO, Rooke GA, Wu RS, Bishop MJ. Comparison of the effects of etomidate, propofol, and thiopental on respiratory resistance after tracheal intubation. Anesthesiology. 1996;84(6):1307-11.

28. Kox WJ, von Heymann C, Heinze J, Prichep LS, John ER, Rundshagen I. Electroencephalographic mapping during routine clinical practice: cortical arousal during tracheal intubation? Anesthesia and analgesia. 2006;102(3):825-31.

29. Reddy RV, Moorthy SS, Dierdorf SF, Deitch RD, Jr., Link L. Excitatory effects and electroencephalographic correlation of etomidate, thiopental, methohexital, and propofol. Anesthesia and analgesia. 1993;77(5):1008-11.

30. den Brinker M, Joosten KF, Liem O. Adrenal insufficiency in meningococcal sepsis: bioavailable cortisol levels and impact of interleukin-6 levels and intubation with etomidate on adrenal function and mortality. The Journal of clinical endocrinology and metabolism. 2005;90(9):5110-17.

31. Payen JF, Dupuis C, Trouve-Buisson $\mathrm{T}$. Corticosteroid after etomidate in critically ill patients: a randomized controlled trial. Crit Care Med. 2012;40(1):29-35.

32. Rogers R, Wise RG, Painter DJ, Longe SE, Tracey I. An investigation to dissociate the analgesic and anesthetic properties of ketamine using functional magnetic resonance imaging. Anesthesiology. 2004;100(2):292-301.

33. Hanouz JL, Persehaye E, Zhu L. The inotropic and lusitropic effects of ketamine in isolated human atrial myocardium: the effect of adrenoceptor blockade. Anesthesia and analgesia. 2004;99(6):1689-95.

34. Hanouz JL, Zhu L, Persehaye E. Ketamine preconditions isolated human right atrial myocardium: roles of adenosine triphosphatesensitive potassium channels and adrenoceptors. Anesthesiology. 2005;102(6):1190-96.

35. Chen RM, Chen TL, Lin YL, Chen TG, Tai YT. Ketamine reduces nitric oxide biosynthesis in human umbilical vein endothelial cells by down-regulating endothelial nitric oxide synthase expression and intracellular calcium levels. Crit Care Med. 2005;33(5):1044-49.

36. April MD, Arana A, Schauer SG. Ketamine Versus Etomidate and Peri-intubation Hypotension: A National Emergency Airway Registry Study. Academic emergency medicine : official journal of the Society for Academic Emergency Medicine. 2020;27(11):1106-15.

37. Mohr NM, Pape SG, Runde D, Kaji AH, Walls RM, Brown CA, 3rd. Etomidate Use Is Associated With Less Hypotension Than Ketamine for Emergency Department Sepsis Intubations: A NEAR Cohort Study. Academic emergency medicine : official journal of the Society for Academic Emergency Medicine. 2020;27(11):1140-49.

38. Grace RF. The effect of variable-dose diazepam on dreaming and emergence phenomena in 400 cases of ketamine-fentanyl anaesthesia. Anaesthesia. 2003;58(9):904-10.

39. Cornelius BG, Webb E, Cornelius A. Effect of sedative agent selection on morbidity, mortality and length of stay in patients with increase in intracranial pressure. World journal of emergency medicine. 2018;9(4):256-61.

40. Cohen L, Athaide V, Wickham ME, Doyle-Waters MM, Rose NG, Hohl CM. The effect of ketamine on intracranial and cerebral perfusion pressure and health outcomes: a systematic review. Annals of emergency medicine. 2015;65(1):43-51.

41. Veselis RA, Reinsel RA, Feshchenko VA, Johnson $\mathrm{R}$, Jr. Information loss over time defines the memory defect of propofol: a comparative response with thiopental and dexmedetomidine. Anesthesiology. 2004;101(4):831-41.

42. Veselis RA, Feshchenko VA, Reinsel RA, Beattie B, Akhurst TJ. Propofol and thiopental do not interfere with regional cerebral blood flow response at sedative concentrations. Anesthesiology. 2005;102(1):26-34.

43. Kodaka M, Johansen JW, Sebel PS. The influence of gender on loss of consciousness with sevoflurane or propofol. Anesthesia and analgesia. 2005;101(2):377-81.

44. Shangguan WN, Lian Q, Aarons L. Pharmacokinetics of a single bolus of propofol in chinese children of different ages. Anesthesiology. 2006;104(1):27-32.

45. Muñoz HR, Cortínez LI, Ibacache ME, Altermatt FR. Estimation of the plasma effect site equilibration rate constant $(\mathrm{ke} 0)$ of propofol in children using the time 
to peak effect: comparison with adults. Anesthesiology. 2004;101(6):1269-74.

46. Pizov R, Brown RH, Weiss YS. Wheezing during induction of general anesthesia in patients with and without asthma. A randomized, blinded trial. Anesthesiology. 1995;82(5):1111-16.

47. Bein B, Renner J, Caliebe D. Sevoflurane but not propofol preserves myocardial function during minimally invasive direct coronary artery bypass surgery. Anesthesia and analgesia. 2005;100(3):61016.

48. Whyte SD, Booker PD, Buckley DG. The effects of propofol and sevoflurane on the QT interval and transmural dispersion of repolarization in children. Anesthesia and analgesia. 2005;100(1):71-77.
49. Gottschling S, Meyer S, Krenn T. Effects of shortterm propofol administration on pancreatic enzymes and triglyceride levels in children. Anaesthesia. 2005;60(7):660-3.

50. Smischney NJ, Seisa MO, Morrow AS. Effect of Ketamine/Propofol Admixture on Peri-Induction Hemodynamics: A Systematic Review and MetaAnalysis. Anesthesiology research and practice. 2020;2020:9637412.

Cite this article as: Aboshoushah EF, Saddeek AY, Alanazi WM, Alghamdi MA, Alrahwan RA, Khayat NA et al. Comparison of different induction agents used for rapid sequence intubation. Int J Community Med Public Health 2021;8:5069-74. 